\title{
Effects of different treatments on seed germination of Elymus tangutorum infected with Epichloë bromicola
}

\author{
Fang-Shu Cheng ${ }^{1}$, Ren-Hui $\mathrm{Li}^{1}$, Fan $\mathrm{Li}^{1}$, Xiao-Yan $\mathrm{Han}^{1}$ and Qiu-Yan Song ${ }^{1 *}$ \\ ${ }^{1}$ State Key Laboratory of Grassland Agro-ecosystems, Key Laboratory of Grassland Livestock Industry Innovation, Ministry of \\ Agriculture and Rural Affairs, College of Pastoral Agriculture Science and Technology, Lanzhou University, Lanzhou 730020, China
}

\begin{abstract}
The seeds of Elymus tangutorum infected with Epichloë bromicola were treated by soaked, wet-dry cycle, mechanical damage, and fungicide. The germination time and germination rate the seeds were measured. The results showed that each treatment had a certain effect on the germination of $E$. tangutorum infected with Ep. bromicola seeds. The total germination rate: wet-dry cycle (soaked for $6 \mathrm{~h}$, dried for $6 \mathrm{~h})>$ mechanical damage $>$ fungicide (cyclosporin T) immersion $(6 \mathrm{~h})>$ aseptic water soaked $(6 \mathrm{~h})>$ CK can be seen from the experimental data. And wet-dry cycle, aseptic water soaked, and mechanical damage have significant effect on increasing the rate of seeds. Among these methods, the germination rate of the seeds treated by wet-dry cycle method was the highest. The treatment has both chemical and physiological functions, thus improving the germination rate and propagation coefficient of seeds.
\end{abstract}

\section{Introduction}

Elymus tangutorum is a perennial forage of the genus Elymus, which has strong adaptability, drought and cold resistance, not easy lodging, developed root system, fresh and tender grass, high crude protein content and good palatability ${ }^{[1,2]}$. Cattle, sheep and horses all like to eat, especially for large livestock. Grass endophyte had the characteristic of providing various resistance to host plants, such as bacteria resistance, insecticidal property, competitiveness improvement, salt tolerance, drought resistance, cold resistance, et ${ }^{[3]}$. At present, the poisoning of livestock caused by E. tangutorum has not been reported yet, suggesting that $E$. tangutorum was a good forage grass. Under biotic and abiotic stresses, Gramineae plants infected with Epichlö have advantages of rapid growth and resistance to stress, diseases, and animal predation, improving their survival competitiveness and nutrient utilization efficiency compared with non-infected plants ${ }^{[4,5]}$.

The seeds of E. tangutorum infected with Epichloë bromicola germination rate is not high. Therefore, how to effectively improve the seed germination rate of $E$. tangutorum, restore the degraded grassland vegetation community and ecological restoration. It is the difficulty of current theoretical and practical research. In this experiment, different pretreatments such as soaked, wet-dry cycle, fungicide method and mechanical damage method were used to promote seed germination ${ }^{[6,7]}$, shorten the time of germination and improve the survival rate of artificial planting of E. tangutorum. It is expected to provide a scientific basis for the successful seed germination and normal seedling formation of $E$. bromicola, and to provide a reference basis for the popularization of E. bromicola in alpine pastoral areas and the preservation of germplasm resources.

\section{Experimental Section}

\subsection{Germination test}

The seeds were treated with soaked, fungicide (cyclosporine T), dry-wet cycle, and mechanical damage method. The groups without any treatment were used as the blank control. The details are as follows. According to the method of petri dish filter paper, the 50 seeds under different treatments were evenly placed in the petri dish covered with 2 layers of filter paper. The seeds were placed according to the method of $5 \times 10$ in each group and put into the incubator (light for $16 \mathrm{~h}$, no light for $8 \mathrm{~h}$ ). Each treatment had three repetitions.

\subsection{Different treatments}

(1) CK: seeds without any treatment

(2) Soaked: seeds soaked in aseptic water (6h).

(3) Fungicide (cyclosporine T) method: seeds soaked in cyclosporine $\mathrm{T}(0.01 \mathrm{mg} / \mathrm{L})(6 \mathrm{~h})$, and the surface residue washing with aseptic water.

(4) Wet-dry cycle: seeds were treated with moisture absorption (6h) and drying (6h), respectively.

(5) Mechanical injury: cut off a quarter of the

\footnotetext{
*songqy@1zu.edu.cn
} 
endosperm from the seed with a sharp blade.

\subsection{Statistical Data Analysis}

Count the number of seeds germinated every day and calculate the germination rate. The significance was analyzed in SPSS 25 software.

\subsection{0-seed weight}

E. tangutorum infected with Ep. bromicola seeds flattened round or semicircular. 1000-seed weight is an important index to reflect the size and plumpness of seeds. E. tangutorum infected with Ep. bromicola seeds average 1000 -seed weight was $4.649 \mathrm{~g}$.

\section{Results and discussion}

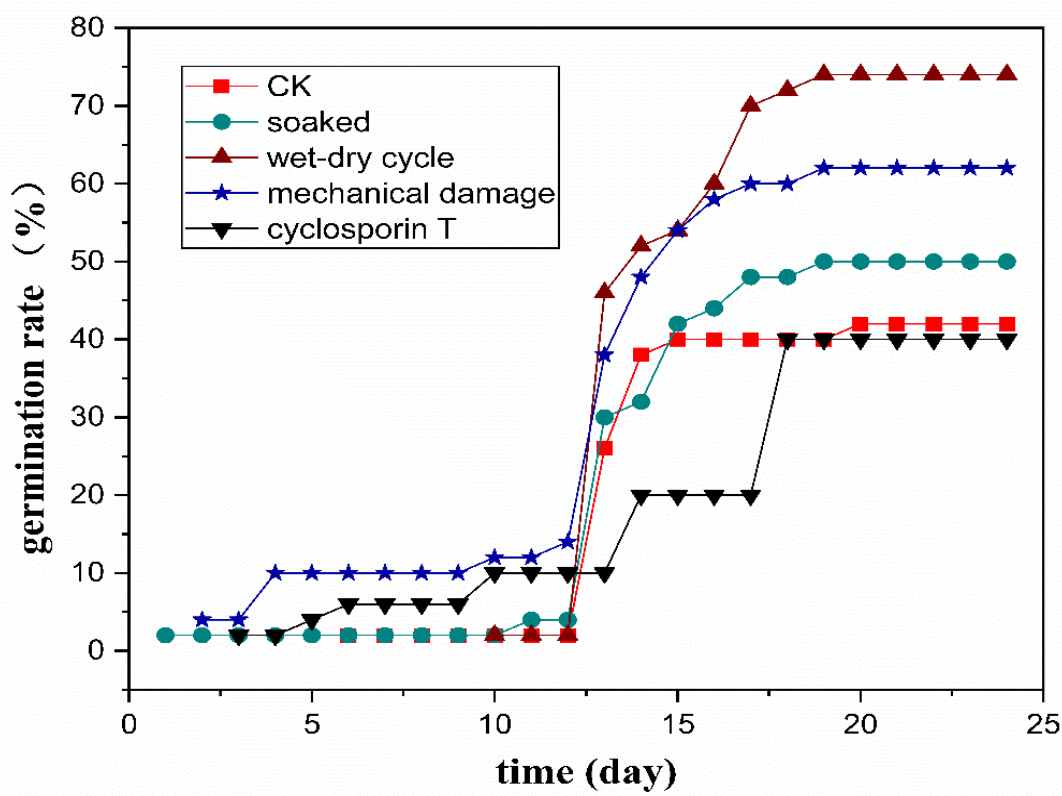

Fig. 1. Effects of different treatments on seed germination of El. Tangutorum

\subsection{Effects of different treatments on seeds germination of E. tangutorum infected with Ep. bromicola}

(1) CK: The blank control group began to germinate on the 6th day after culture, the peak period of germination is $13-15$ days, and the total germination rate is $42 \%$. The results were shown in Fig. 1.

(2) Soaked: Water is one of the important factors affecting seed germination. Only when the seed cells have enough free water can the enzyme be activated to react. The experimental results show that the soaked method can effectively improve the germination rate of $E$. tangutorum infected with Ep. bromicola. The seeds soaked for $6 \mathrm{~h}$ in the treatment group began to germinate on the first day, the peak germination period of aseptic water-soaked seeds was 15-17 days, and the average germination process was 19 days, and the total germination rate is $50 \%$.

(3) Fungicide (cyclosporine T) method: $E$. tangutorum infected with Ep. bromicola produced a polypeptide compound, cyclosporine $\mathrm{T}$, which had excellent antifungal activity at low concentration. At present, it is not known whether cyclosporine $\mathrm{T}$ can play a positive role in raising the germination rate of $E$. tangutorum infected with Ep. bromicola. In this experiment, the results showed that the germination rate of seeds soaked with cyclosporine $\mathrm{T}$ of $0.1 \mathrm{mg} / \mathrm{L}$ for 6 hours did not increase significantly.

(4) wet-dry cycle: The effect of moisture absorption and re-drying is the alternating treatment of wet and dry before sowing, and some people call this treatment a method to strengthen the "exercise" of seeds ${ }^{[8,9]}$. $E$. tangutorum infected with Ep. bromicola seeds treated by wet-dry cycle method the germination rate reached $74 \%$. The whole germination period is very short, with an average of 10 days.

(5) Mechanical injury: Seeds need to break their dormancy if they want to germinate. The poor air permeability of gramineous forage seeds is due to the fact that there is a glume wrapped around the seeds, which leads to the insufficient supply of oxygen to embryos. Ventilation is very necessary for the production of gibberellin in seeds, and the gibberellins produced can increase the activities of amylase, peroxidase and phospholipase in seeds, thus promoting seed ripening, relieving seed dormancy and strengthening seed germination. The treatment of seeds by mechanical damage can break the dormancy of seeds to a great 
extent. The germination rate is higher than the blank control group, with an average germination rate of $62 \%$.

\subsection{Significance test of differences among different treatments}

As shown in Table 1., the germination rates of the four treatments that the seeds were treated with soaked, wet-dry cycle and mechanical damage method were higher than those of the blank control at 0.05 level. Multiple comparisons showed that wet-dry cycle method could significantly improve the germination rate of seeds, which was significantly higher than that of other treatment. The effect of seed soaked with cyclosporine $\mathrm{T}$ was similar to that of seed soaked with aseptic water and the difference was not significant.

Table 1. Significant differences of germination rate of $E$. tangutorum infected with Ep. bromicola seeds treated with different treatments

\begin{tabular}{|l|l|l|}
\hline seeds treatment & $\begin{array}{l}\text { seeds } \\
\text { germination } \\
\text { rate } \mathbf{( 1 0 0 \% )}\end{array}$ & $\begin{array}{l}\text { significant } \\
\text { difference } \\
(\mathbf{0 . 0 5})\end{array}$ \\
\hline ck & 42 & $\mathrm{~d}$ \\
\hline soaked & 50 & $\mathrm{c}$ \\
\hline cyclosporine T & 40 & $\mathrm{~d}$ \\
\hline wet-dry cycle & 74 & $\mathrm{a}$ \\
\hline mechanical injury & 62 & $\mathrm{~b}$ \\
\hline
\end{tabular}

\section{Conclusion}

The experimental results show that the most obvious improvement of the germination rate was the wet-dry cycle method in which the seeds were soaked for $6 \mathrm{~h}$, and then the seeds were re-dried for $6 \mathrm{~h}$. Soaked seeds in aseptic water and mechanical damage can also increase the germination rate, but soaked seeds with cyclosporine $\mathrm{T}$ has no obvious effect. Therefore, the results provided a theoretical basis for the field cultivation of $E$. tangutorum infected with Ep. bromicola.

The vigor of all seeds has been a foregone conclusion since it was harvested, and as the seeds are stored longer, their vigor levels only decrease and cannot be improved (except for post-ripening and dormant seeds). Usually in production, the method of increasing the amount of sowing is usually used to improve the emergence rate of seedlings in the field. In this paper, the experiment of $E$. tangutorum infected with Ep. bromicola showed that the combined treatment of cytokinin and moisture absorption and re-drying before sowing could effectively improve the germination performance of seeds, improve the germination rate and stress resistance of seeds, that is to say, improve the vigor level of seeds as a whole. The method of increasing the amount of sowing cannot achieve this effect.

\section{Aknowledgement}

This project was supported financially by the National Natural Science Foundation of China (31901388), the Special Administrative Region (SAR) of Herbal Medicinein Gansu Province (18JR4RA003), the Fundamenta Research Funds for the Central Universities (lzujbky-2020-20)

\section{References}

1. M. L. Jing, Y. S. Ma, H. M. Li, J. L. Wang. Mitochondrial DNA B. Characterization of the complete plastome of Elymus tangutorum (Poaceae: Triticeae). 4, 3356-3357 (2019)

2. Q. Y. Song, Z. B. Nan, K. Gao, H. Song, P. Tian, X. X. Zhang, C. J. Li, W. B. Xu, X. Z. Li. J Agr Food Chem. Antifungal, Phytotoxic, and Cytotoxic Activities of Metabolites from Epichloe bromicola, a Fungus Obtained from Elymus tangutorum Grass. 63, 8787-8792 (2015)

3. J. J. Fu, J. Liu, L. Y. Yang, Y. J. Miao, Y. F. Xu. J Agr Sci Tech-Iran. Effects of Low Temperature on Seed Germination, Early Seedling Growth and Antioxidant Systems of the Wild Elymus nutans Griseb. 19, 1113-1125 (2017)

4. J. F. Wang, W. P. Hou, M. J. Christensen, C. Xia, T. Chen, Z. X. Zhang, Z. B. Nan. Sci China Life Sci. The fungal endophyte Epichloe gansuensis increases NaCl-tolerance in Achnatherum inebrians through enhancing the activity of plasma membrane H+-ATPase and glucose-6-phosphate dehydrogenase. 10.1007/s11427-020-1674-y (2020)

5. C. Xia, N. N. Li, Y. W. Zhang, C. J. Li, X. X. Zhang, Z. B. Nan. Plant Dis. Role of Epichloe Endophytes in Defense Responses of Cool-Season Grasses to Pathogens: A Review. 102, 2061-2073 （2018）

6. B. L. Deng, S. Tian, S. P. Li, M. X. Guo, H. X. Liu, Y. Y. Li, Q. J. Wang, X. S. Zhao. Food Chem. A simple, rapid and efficient method for essential element supplementation based on seed germination. 325, (2020)

7. X. Z. Li, M. L. Song, X. Yao, Q. Chai, W. R. Simpson, C. J. Li, Z. B. Nan. Front Microbiol. The Effect of Seed-Borne Fungi and Epichloe Endophyte on Seed Germination and Biomass of Elymus sibiricus. 8, 2488 (2017)

8. K. V. Supraja, B. Behera, P. Balasubramanian. Ind Crop Prod. Efficacy of microalgal extracts as biostimulants through seed treatment and foliar spray for tomato cultivation. 151, (2020)

9. Z. F. Wang, C. J. Li, J. White. J Agron Crop Sci. Effects of Epichloe endophyte infection on growth, physiological properties and seed germination of wild barley under saline conditions. 206, 43-51 (2020) 